\section{KOMPASS}

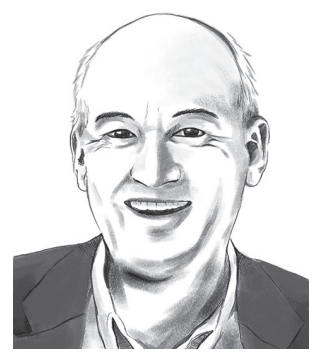

Harald Walach

Poznań/Berlin,

Polen/Deutschland

Mein Kollege an der Universität Witten-Herdecke ist Mathematiker und ein ziemlicher Witzbold. An seiner Tür sind allerhand lustige Karten und Sprüche angebracht. Einer lautet: «In der Nacht, wenn alle schlafen und es niemand sieht, teile ich heimlich durch Null.» Aber durch Null teilen geht nicht. Absolut verboten. Sinnlosigkeit schlechthin, könnte man sagen. Lassen Sie mich diese Sinnlosigkeit schlechthin ausloten.

Die Einführung der Null in die Mathematik war einer der größten Fortschritte. Denn sie erlaubt das Zählen und Rechnen, wie wir es kennen und wie es Grundlage unseres gesamten wissenschaftlichen Fortschrittes geworden ist. Zumindest soweit Mathematik daran beteiligt ist, und wo ist sie das nicht.

Nehmen wir die Zahl 1027. Das ist eine 10 mit 27 Nullen (!) hintendran. $10^{27}$ m markiert die Größe unseres sichtbaren Universums. Oder $10^{-33}$, eine Null mit 33 Nullen (!) hinter dem Komma. 10-33 m markiert die kleinste Größe, die Planck-Skala, die Grenze der Anwendbarkeit der bekannten Gesetze der Physik. Zwischen $10^{27} \mathrm{~m}$ und $10^{-33} \mathrm{~m}$ sind alle Dimensionen unserer Welt aufgespannt. Immer fein mit Nullen, mehr oder weniger, markiert [1].

Die Medizin beschäftigt sich ja hauptsächlich mit dem, man könnte sagen, materiellen Aspekt des Menschen. Mit dem Körper,

Karger Kompass Pneumol 2019;7:178

DOI: 10.1159/000500386

\title{
Teilen durch Null
}

seinen Organen, seinen Zellen, deren Organellen und Makromolekülen, und reicht mit allen möglichen Proben bereits in den Nanobereich der Materie. Betrachten wir also mal diese Materie. Eigentlich sind 99,9999999999999\% davon «Nichts», Leere. Ein Atomkern ist so ähnlich wie ein Basketball in einem Spielfeld und in etwa $3 \mathrm{~km}$ Entfernung kommt dann das Elektron. Es hat nur deswegen die gefühlte «Dichte» und Widerständigkeit eines Körpers, weil die Elektronen dazwischen ausgeschmiert sind und mit einer Frequenz von ungefähr $10^{12}$ $\mathrm{Hz}$ schwingen. Witzig, oder?

$$
\begin{aligned}
& \text { Wieder ist das «Nichts», } \\
& \text { das nicht Sicht- und Greifbare, } \\
& \text { das Entscheidende. }
\end{aligned}
$$

Nun, wenn es zu unserer bekannten Materie kommt, dann kennen wir eigentlich nur 5\% davon. Soviel macht alle bekannte Energie und Materie im Universum aus. 95\% aller Energie und Materie sind unbekannt. Ungefähr 23\% dunkle Materie, von der man annimmt, sie wäre für Gravitationskräfte zuständig, und etwa 72\% dunkle Energie, die eher expansiv zu wirken scheint [2]. Wieder ist das «Nichts», das nicht Sicht- und Greifbare, das Entscheidende.

Viele denken, wir seien knapp davor, alles zu verstehen. Wie der Körper und der Geist funktionieren, wie man Krankheiten heilt.
Möglicherweise sind wir damit aber genauso am Anfang wie mit dem Verständnis unseres Kosmos. Weil wir nämlich die Aufmerksamkeit zu wenig auf das «Dazwischen» lenken, das vermeintlich nicht Greifbare und Unsichtbare. Möglicherweise wäre eine Medizin, die sich nicht nur auf das materiell Sichtbare stützt, sogar eine Option für die Zukunft?

Ich habe einmal eine Heilerin interviewt, Ursula Kress, die vor Kurzem verstorben ist. Sie behandelte vor allem Kinder mit verkrüppelten Gliedmaßen mit großem Erfolg [3]. Ich fragte sie, was sie da täte. Sie sagte: «lch stelle mir vor, wie es sein soll, und genauso wird es dann.» Teilen durch Null ist das. Und anders als beim Computer, scheint es bei uns Menschen zu funktionieren.

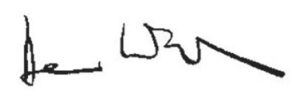

Prof. Dr. Dr. phil. Harald Walach

\section{Literatur}

1 Carr BR. Hyperspatial models of matter and mind; in Kelly EF, Crabtree A, Marshall P (eds): Beyond Physicalism: Toward Reconciliatoin of Science and Spirituality, Lanham, MD, Rowman \& Littlefield, 2015, pp 227-273.

2 Currivan J: The Cosmic Hologram: In-Formation at the Center of Creation, Rochester, Inner Traditions, 2017.

3 Kress U: Heilende Hände: Der Heilmagnetismus, Hennef, Verlag Uta Halft, 1986.

\section{KARGER}

Fax +4976145207 14 information@karger.com www.karger.com

\section{(c) 2019 S. Karger GmbH, Freiburg}

Accessible online at: www.karger.com/kkp
Prof. Dr. Dr. phil. Harald Walach

CHS Institut

Schönwalder Straße 17, 13347 Berlin, Deutschland

hw@chs-institute.org 